UDK $577.1: 61$

ISSN 1452-8258

J Med Biochem 38: 407-417, 2019

\title{
PREDICTIVE VALUES OF SERUM URIC ACID AND ALANINE-AMINOTRANSFERASE FOR FATTY LIVER INDEX IN MONTENEGRIN POPULATION
}

\author{
PREDIKTIVNE VREDNOSTI MOKRAĆNE KISELINE I ALANIN-AMINOTRANSFERAZE \\ U CILJU DIJAGNOSTIKOVANJA MASNE JETRE U CRNOGORSKOJ POPULACIJI
}

\author{
Aleksandra Klisic ${ }^{1}$, Nebojsa Kavaric ${ }^{1}$, Ana Ninic ${ }^{2}$ \\ ${ }^{1}$ Primary Health Care Center, Podgorica, Montenegro \\ ${ }^{2}$ Department for Medical Biochemistry, University of Belgrade - Faculty of Pharmacy, Belgrade, Serbia
}

\begin{abstract}
Summary
Background: Alanine-aminotransferase (ALT) and uric acid cut-off levels used in non-alcoholic fatty liver disease (NAFLD) diagnosis are advised to be lowered. Due to contradictory results on the utility of both these biomarkers for NAFLD screening, we aimed to determine their cut-off levels that can be applied to Montenegrin population with the fatty liver disease.

Methods: A total of 771 volunteers were enrolled. A fatty liver index (FLI) score $\geq 60$ was used as proxy of NAFLD. The receiver operating characteristic curve analysis with the area under the curve (AUC) was used to determine the cutoff values of ALT and uric acid associated with FLI $\geq 60$.

Results: ALT was independent predictor of FLI in both men and women, whereas serum uric acid was its independent predictor only in women. Lower cut-off levels of ALT are associated with the increased prevalence of NAFLD [i.e., ALT was $19 \mathrm{IU} / \mathrm{L}$ (AUC $=0.746$, sensitivity $63 \%$, specificity $72 \%, \mathrm{P}<0.001$ ) in women and $22 \mathrm{IU} / \mathrm{L}$ ( $\mathrm{AUC}=0.804$, sensitivity $61 \%$, specificity $95 \%, P<0.001$ ) in men]. The cut-off value for uric acid was $274 \mu \mathrm{mol} / \mathrm{L}$ (AUC=0.821, sensitivity $68 \%$, specificity $82 \%, \mathrm{P}<0.001$ ) in women.

Conclusions: Lower cut-off levels of ALT in both genders, and serum uric acid in females, can be reliable predictors of the FLI.
\end{abstract}

Keywords: fatty liver, hyperuricemia, inflammation, transaminases, obesity

Address for correspondence:

Aleksandra Klisic, MD, PhD

Center for Laboratory Diagnostics, Primary Health Care Center, Trg Nikole Kovacevica 6, 81000 Podgorica, Montenegro

Phone and Fax: +382 20481999

e-mail: aleksandranklisic@gmail.com

\section{Kratak sadržaj}

Uvod: Preporuka skorijih studija je da cut-off vrednosti alanin-aminotransferaze (ALT) i mokraćne kiseline budu niže u cilju dijagnostikovanja nealkoholne steatoze jetre. S obzirom na postojanje oprečnih rezultata kada su u pitanju ovi biomarkeri u cilju skrininga na nealkoholnu steatozu jetre, cilj ove studije je bio da se odrede cut-off vrednosti koje bi mogle biti primenjive u crnogorskoj populaciji za dijagnostiku ovog poremećaja.

Metode: Istraživanje je obuhvatilo ukupno 771 dobrovoljca. Indeks masne jetre (engl. fatty liver index-FLI) $\geq 60$ je korišten za dijagnostikovanje nealkoholne steatoze jetre. ROC analiza i površina ispod krive (engl. area under the curve-AUC) su korišteni za određivanje cut-off vrednosti ALT i mokraćne kiseline, koji su povezani sa FLI $\geq 60$.

Rezultati: ALT je nezavisan prediktor FLI kod oba pola, dok je mokraćna kiselina nezavisan prediktor masne jetre samo kod žena. Takođe, niže cut-off vrednosti ALT su povezane sa većom prevalencom pojave masne jetre [za ALT kod žena vrednost je $19 \mathrm{IU} / \mathrm{L}$ ( $\mathrm{AUC}=0,746$, senzitivnost 63\%, specifičnost $72 \%, P<0,001)$, a kod muškaraca je $22 \mathrm{IU} / \mathrm{L}$ (AUC $=0,804$, senzitivnost $61 \%$, specifičnost $95 \%, P<0,001$ )]. Cut-off vrednost za mokraćnu kiselinu za pojavu masne jetre kod žena je $274 \mu \mathrm{mol} / \mathrm{L}(\mathrm{AUC}=0,821$, senzitivnost $68 \%$, specifičnost $82 \%, \mathrm{P}<0,001)$.

Zaključak: Niže cut-off vrednosti ALT kod oba pola, kao i niže vrednosti mokraćne kiseline kod žena, mogu biti pouzdani prediktori za pojavu masne jetre.

Ključne reči: masna jetra, hiperurikemija, inflamacija, transaminaze, gojaznost 


\section{Introduction}

It is widely recognized that non-alcoholic fatty liver disease (NAFLD) represents the commonest manifestation of chronic liver diseases (1). Its prevalence is rising along with the growing proportion of obesity and diabetes mellitus type 2 (DM2) worldwide. Although it looks like a benign condition, without any symptoms, during a certain time NAFLD increases the risk of cirrhosis and hepatocellular carcinoma (1). Importantly, it is an early predictor of diabetic complications and cardiovascular disease (CVD) $(2,3)$.

Liver biopsy is established as the gold standard for diagnosis of hepatic steatosis (4). However, due to its invasive diagnostic nature it is not suitable procedure in routine everyday praxis. Therefore, it has been replaced with abdominal ultrasonography, as the commonest technique for NAFLD assessment in clinical trials. In line with this, Bedogni et al. (5) derived fatty liver index (FLI), an algorithm based on body mass index (BMI), waist circumference (WC), triglycerides (TG) and gamma-glutamyl transferase (GGT), as a simple and accurate predictor of NAFLD. An FLI score $\geq 60$ has been shown to have good sensitivity and specificity for NAFLD when diagnosed by abdominal ultrasonography, thus making it suitable for assessment hepatic steatosis in general population $(5,6)$.

Alanine-aminotransferase $(\operatorname{ALT})(7,8)$, and uric acid $(9,10)$, are shown to be independently associated with NAFLD. On the other hand, some studies advised that the ALT cut-off level used in NAFLD diagnosis should be revised and lowered (11, 12 ), since a threshold of $40 \mathrm{IU} / \mathrm{L}$ is commonly used in clinical practice (12). However, even with lower cutoff levels, ALT was shown to be a poor marker of NAFLD in some studies $(8,12,13)$.

Similarly, discrepant results were observed when examining uric acid in relation to NAFLD, especially focusing on sex-specific differences and obesity status (14-17).

To our knowledge, there are no data on the prevalence of fatty liver in general population in Montenegro. Considering the high prevalence of overweight/obesity (18) and DM2 (19) in Montenegro, the early diagnosis of NAFLD is of great importance. Due to discrepant results on the utility of both these biomarkers for NAFLD screening, we aimed to determine their cut-off levels that can be applied to Montenegrin population with fatty liver disease.

\section{Materials and Methods}

\section{Study population}

Out of the total number of 1000 participants (397 men and 603 women) who were screened, 771 of them met the inclusion criteria in the current cross- sectional study (249 men and 522 women). Participants were sequentially recruited in the Primary Health Care Centre in Podgorica, Montenegro, during their routine check-up in a period from October 2012 to May 2016. Subjects older than 18 years of age were included in the study. Participants were regarded to have DM2 if they exibited $\mathrm{HbA} 1 \mathrm{c} \geq 6.5 \%$ measured on two different occasions, or with at least two measurements of fasting glucose levels $\geq 7.0 \mathrm{mmol} / \mathrm{L}$, or with a random plasma glucose level of $\geq 11.1$ $\mathrm{mmol} / \mathrm{L}$, or a plasma glucose level $\geq 11.1 \mathrm{mmol} / \mathrm{L} 2$ $\mathrm{h}$ after an oral glucose tolerance test. Also, participants were regarded to have DM2 if they self-reported DM2, as well as if they were treated with oral hypoglycemic agents or insulin, as described elsewhere (20).

Participants were excluded from the research if they had liver disease other than NAFLD, malignant diseases, renal dysfunction, cardiovascular diseases, ethanol consumption $>20 \mathrm{~g} /$ day, type 1 diabetes mellitus, high sensitivity $\mathrm{C}$-reactive protein levels (hsCRP) $>10 \mathrm{mg} / \mathrm{L}$, younger than 18 years of age, pregnancy.

Written informed consent was provided by each participant and all procedures performed in the current research were in accordance with the standards of the Ethical Committee of Primary Health Care Centre in Podgorica, Montenegro and with the Declaration of Helsinki.

\section{Anthropometric and blood pressure measurements}

Basic anthropometric measurements, systolic blood pressure (SBP) and diastolic blood pressure (DBP) were measured as described previously (21).

\section{Biochemical analyses}

The blood samples were taken in the morning, between 7 and 10 o'clock, after at least 8 hours of fasting. Serum levels of glucose, total cholesterol, high density lipoprotein cholesterol (HDL-c), low density lipoprotein cholesterol (LDL-c), TG, creatinine, uric acid, total bilirubin, aspartate aminotransferase (AST), ALT and GGT, were measured using standardized enzymatic procedures, spectrophotometrically (Roche Cobas 400, Mannheim, Germany). Serum hsCRP levels were determined using a nephelometric assay (Behring Nephelometer Analyzer, Marburg, Germany).

\section{Assessment of non-alcoholic fatty liver disease}

Non-alcoholic fatty liver disease is assessed by FLI, using the following algorithm (5):

$\mathrm{FLI}=\left(\mathrm{e}^{0.953 \times \text { loge (triglycerides) }}+0.139 \times \mathrm{BMI}+0.718 \times\right.$ loge $(\mathrm{GGT})+0.053 \times$ waist circumference -15.745$) /\left(1+\mathrm{e}^{0.953 \times \text { loge }}\right.$ (triglycerides) $+0.139 \times \mathrm{BMI}+0.718 \times$ loge $(\mathrm{GGT})+0.053 \times$ waist circumference -15.745$) \times 100$. 
A FLI score $\geq 60$ has been shown to have good sensitivity and specificity, thus making it a convenient proxy of NAFLD. The 2016 EASL-EASD-EASO NAFLD guidelines are based on the recommendations on the usage of the serum biomarkers as endorsed diagnostic tool with the FLI as one of the best validated steatosis scores for screening studies in large population samples (22).

\section{Statistical analysis}

The parameter distribution was tested by the Kolmogorov Smirnov test and the data were presented as median (interquartile range). Categorical data were presented as relative frequencies and compared by Chi-square test for contingency tables. Kruskal-Wallis test was employed for comparisons between three groups of patients based on FLI separately in men and women. Correlations between FLI and clinical parameters were tested by Spearman's correlation analysis. Multivariate logistic regression analysis was used to find models consisting of clinical parameters (predictors, independent variables) which significantly influence the variability in the FLI, as dependent variable. The group of patients with $\mathrm{FLI}<30$ was coded as 0 , while the group of patients with $\mathrm{FLI} \geq 60$ was coded as 1 . Variables in the FLI equation (i.e. BMI, WC, TG and
GGT) were excluded from multivariate analyses. Results are presented by odds ratio (OR) with 95\% confidence intervals $(\mathrm{Cl})$. The explained variation in FLI was given by Nagelkerke $\mathrm{R}^{2}$ value for single predictors and models. The Hosmer and Lemeshow test was used to examine whether there was a linear relationship between the independent variables and the log odds of the dependent variable. The clinical accuracy of the examined parameters and models were assessed by using receiver operating characteristic (ROC) curve analysis. The same analysis was also used to determine the cut-off values of the ALT and uric acid associated with the increase in the prevalence of the fatty liver disease (i.e., $\mathrm{FLI} \geq 60$ ) in both genders. The area under the ROC curve (AUC) between 0.5 and 0.7 suggested the low accuracy of diagnostic test; between 0.7 and 0.8 satisfactory accuracy, between 0.8 and 0.9 good accuracy, while AUC higher than 0.9 suggested the excellent accuracy of diagnostic test (23). Statistical analyses were performed with IBM ${ }^{\circledR}$ SPSS ${ }^{\circledR}$ Statistics version 22 software (USA). In all the analyses, values were considered statistically significant at $\mathrm{P}<0.05$.

\section{Results}

Table I indicates the general characteristics of men according to FLI. As expected, all three FLI

Table I General clinical data of study men's population according to FLI.

\begin{tabular}{|l|c|c|c|c|}
\hline & $\begin{array}{c}\text { First FLI group } \\
(\mathrm{FLI}<30)\end{array}$ & $\begin{array}{c}\text { Second FLI group } \\
(\mathrm{FLI} \geq 30,<60)\end{array}$ & $\begin{array}{c}\text { Third FLI group } \\
(\mathrm{FLI} \geq 60)\end{array}$ & $P$ \\
\hline $\mathrm{N}$ & 28 & 66 & 155 & \\
\hline Age, years & $\begin{array}{c}63.00 \\
(53.00-71.00)\end{array}$ & $\begin{array}{c}63.00 \\
(52.50-72.00)\end{array}$ & $\begin{array}{c}61.00 \\
(53.00-67.00)\end{array}$ & 0.253 \\
\hline BMI, $\mathrm{kg} / \mathrm{m}^{2}$ & $\begin{array}{c}24.08 \\
(23.15-25.25)\end{array}$ & $\begin{array}{c}26.60 \\
(264.98-27.70)^{\mathrm{a}}\end{array}$ & $\begin{array}{c}30.67 \\
28.71-33.00)^{\mathrm{a}, \mathrm{b}}\end{array}$ & $<0.001$ \\
\hline WC, $\mathrm{cm}$ & 90.00 & 98.00 & 110.00 \\
$(85.00-85.00)$ & $(96.00-101.00)^{\mathrm{a}}$ & $(104.00-116.00)^{\mathrm{a}, \mathrm{b}}$ & $<0.001$ \\
\hline SBP, $\mathrm{mmHg}$ & $\begin{array}{c}130.00 \\
(126.00-136.00)\end{array}$ & $(127.00-139.00)^{\mathrm{a}}$ & $(126.00-144.00)^{\mathrm{a}}$ & 0.519 \\
\hline DBP, mmHg & 80.00 & 81.00 & 80.00 & 0.0030 \\
\hline Smokers, $\mathrm{n}(\%)$ & $(70.00-90.00)$ & $(76.00-87.00)^{\mathrm{a}}$ & $(74.00-88.00)^{\mathrm{a}}$ & 0.730 \\
\hline Antihyperglycemics, $\mathrm{n}(\%)$ & $5(18 \%)$ & $20(30 \%)$ & $41(26 \%)$ & 0.800 \\
\hline Insulin, $\mathrm{n}$ (\%) & $11(39 \%)$ & $28(42 \%)$ & $89(57 \%)$ & 0.065 \\
\hline Antihyperlipidemics, $\mathrm{n}(\%)$ & $5(18 \%)$ & $10(15 \%)$ & $28(18 \%)$ & 0.643 \\
\hline Antihypertensives, $\mathrm{n}(\%)$ & $11(39 \%)$ & $16(24 \%)$ & $65(42 \%)$ & 0.012 \\
\hline Type 2 diabetes mellitus, $\mathrm{n}(\%)$ & $13(46 \%)$ & $41(62 \%)$ & $111(72 \%)$ & 0.141 \\
\hline
\end{tabular}

Data are presented as median (interquartile range) and compared by Kruskal-Wallis with post hoc test.

a - significantly different from the first FLI group, $\mathrm{p}<0.05$

$b$ - significantly different from the second FLI group, $\mathrm{p}<0.05$

BMI - Body mass index; WC - Waist circumference; SBP - Systolic blood pressure; DBP - Diastolic blood pressure 
Table II General clinical data of study women's population according to FLI.

\begin{tabular}{|c|c|c|c|c|}
\hline & $\begin{array}{l}\text { First FLI group } \\
(F L I<30)\end{array}$ & $\begin{array}{l}\text { Second FLI group } \\
(F L I \geq 30,<60)\end{array}$ & $\begin{array}{l}\text { Third FLI group } \\
\quad(F L I \geq 60)\end{array}$ & $P$ \\
\hline $\mathrm{N}$ & 186 & 137 & 199 & $<0.001$ \\
\hline Age, years & $56.00(49.00-61.00)$ & $60.00(55.00-67.00)^{a}$ & $62.00(56.00-68.00)^{a}$ & $<0.001$ \\
\hline $\mathrm{BMI}, \mathrm{kg} / \mathrm{m}^{2}$ & $23.59(21.50-25.07)$ & $27.78(26.13-29.40)^{a}$ & $32.24(30.18-35.54)^{a, b}$ & $<0.001$ \\
\hline $\mathrm{WC}, \mathrm{cm}$ & $82.00(77.00-88.00)$ & $95.00(92.00-99.00)^{a}$ & $107.00(100.00-112.00)^{a, b}$ & $<0.001$ \\
\hline $\mathrm{SBP}, \mathrm{mmHg}$ & 129.00 (115.00-140.00) & $136.00(127.00-147.00)^{a}$ & $135.00(126.00-146.00)^{a}$ & $<0.001$ \\
\hline $\mathrm{DBP}, \mathrm{mmHg}$ & $78.00(70.00-90.00)$ & $85.00(76.00-94.00)^{a}$ & $81.00(76.00-90.00)^{a}$ & 0.001 \\
\hline Smokers, n (\%) & 27 (15\%) & 19 (14\%) & 25 (13\%) & 0.800 \\
\hline $\begin{array}{l}\text { Antihyperglycemics, } \\
\mathrm{n}(\%)\end{array}$ & $9(5 \%)$ & $28(20 \%)$ & 87 (44\%) & $<0.001$ \\
\hline Insulin, n (\%) & $2(1 \%)$ & $7(5 \%)$ & $14(7 \%)$ & 0.016 \\
\hline $\begin{array}{l}\text { Antihyperlipidemics, } \\
\mathrm{n}(\%)\end{array}$ & 22 (12\%) & 27 (20\%) & $66(33 \%)$ & $<0.001$ \\
\hline $\begin{array}{l}\text { Antihypertensives, } \\
\mathrm{n}(\%)\end{array}$ & $38(20 \%)$ & 56 (41\%) & 134 (67\%) & $<0.001$ \\
\hline $\begin{array}{l}\text { Type } 2 \text { diabetes } \\
\text { mellitus, } \mathrm{n}(\%)\end{array}$ & $10(5 \%)$ & 30 (22\%) & 101 (51\%) & $<0.001$ \\
\hline
\end{tabular}

Data are presented as median (interquartile range) and compared by Kruskal-Wallis with post hoc test

$a$ - significantly different from the first FLI group, $\mathrm{p}<0.05$; $\mathrm{b}$ - significantly different from the second FLI group, $\mathrm{p}<0.05$

BMI - Body mass index; WC - Waist circumference; SBP - Systolic blood pressure; DBP - Diastolic blood pressure

Table III Biochemical analysis of study men's population according to FLI.

\begin{tabular}{|l|c|c|c|c|}
\hline & $\begin{array}{c}\text { First FLI group } \\
(\text { FLI }<30)\end{array}$ & $\begin{array}{c}\text { Second FLI group } \\
(\text { FLI } \geq 30,<60)\end{array}$ & $\begin{array}{c}\text { Third FLI group } \\
(\text { FLI 60) }\end{array}$ & P \\
\hline Glucose, mmol/L & $5.90(5.50-6.30)$ & $6.10(5.40-7.40)$ & $6.70(5.60-8.67)^{a, b}$ & 0.008 \\
\hline Total cholesterol, mmol/L & $4.44(4.12-5.29)$ & $5.09(4.60-5.78)^{a}$ & $5.22(4.57-6.02)^{a}$ & 0.020 \\
\hline HDL-c, mmol/L & $1.52(1.16-1.71)$ & $1.27(1.03-1.61)$ & $1.01(0.88-1.26)^{a, b}$ & $<0.001$ \\
\hline LDL-c, mmol/L & $2.82(1.82-3.39)$ & $2.96(2.68-3.88)$ & $3.20(2.49-3.83)$ & 0.055 \\
\hline TG, mmol/L & $1.00(0.84-1.26)$ & $1.48(1.03-1.80)^{a}$ & $2.08(1.66-2.73)^{a, b}$ & $<0.001$ \\
\hline AST, IU/L & $18.00(16.00-21.00)$ & $21.00(18.00-24.00)^{a}$ & $20.00(18.00-25.00)^{a, b}$ & 0.219 \\
\hline ALT, IU/L & $16.50(14.00-20.00)$ & $22.00(18.00-28.00)^{a}$ & $24.00(17.00-30.00)^{a, b}$ & $<0.001$ \\
\hline GGT, IU/L & $13.00(11.00-17.00)$ & $18.00(14.00-25.00)^{a}$ & $27.00(20.00-37.00)^{a, b}$ & $<0.001$ \\
\hline Uric acid, $\mu \mathrm{mol} / \mathrm{L}$ & $313.00(250.00-366.00)$ & $300.00(262.00-364.00)$ & $333.00(283.00-382.00)$ & 0.084 \\
\hline Total bilirubin, $\mu \mathrm{mol} / \mathrm{L}$ & $9.25(5.50-12.70)$ & $8.70(6.40-11.84)$ & $8.60(5.95-12.07)$ & 0.978 \\
\hline HsCRP, mg/L & $0.73(0.38-1.58)$ & $0.83(0.48-1.66)$ & $1.53(0.84-3.27)^{\mathrm{a}, \mathrm{b}}$ & $<0.001$ \\
\hline Creatinine, $\mu \mathrm{mol} / \mathrm{L}$ & $86.00(80.00-94.00)$ & $83.00(75.00-96.00)$ & $84.00(74.00-97.00)$ & 0.823 \\
\hline FLI & $22.00(15.00-26.00)$ & $48.00(40.00-54.00)^{a}$ & $85.00(73.00-92.00)^{\mathrm{a}, \mathrm{b}}$ & $<0.001$ \\
\hline
\end{tabular}

Data are presented as median (interquartile range) and compared by Kruskal-Wallis with post hoc test.

$a$ - significantly different from the first FLI group, $\mathrm{p}<0.05 ; \mathrm{b}$ - significantly different from the second FLI group, $\mathrm{p}<0.05$

HDL-c-High density lipoprotein cholesterol; LDL-c-Low density lipoprotein cholesterol; TG - Triglycerides; AST - Aspartate aminotransferase; ALT

- Alanine aminotransferase; GGT - Gamma-glutamyl transferase; HsCRP - High-sensitivity C-reactive protein; FLI - Fatty liver index 
Table IV Biochemical analysis of study women's population according to FLI.

\begin{tabular}{|c|c|c|c|c|}
\hline & $\begin{array}{l}\text { First FLI group } \\
\quad(\mathrm{FLI}<30)\end{array}$ & $\begin{array}{l}\text { Second FLI group } \\
(F L I \geq 30,<60)\end{array}$ & $\begin{array}{l}\text { Third FLI group } \\
\quad(F L I \geq 60)\end{array}$ & $P$ \\
\hline Glucose, mmol/L & $5.30(5.00-5.70)$ & $5.60(5.10-6.20)^{a}$ & $6.50(5.60-7.70)^{a, b}$ & $<0.001$ \\
\hline Total cholesterol, mmol/L & $5.80(4.96-6.66)$ & $6.02(5.25-6.71)$ & $6.13(5.20-7.06)^{a}$ & 0.022 \\
\hline $\mathrm{HDL}-\mathrm{c}, \mathrm{mmol} / \mathrm{L}$ & $1.76(1.48-2.02)$ & $1.56(1.28-1.75)^{a}$ & $1.27(1.10-1.45)^{a, b}$ & $<0.001$ \\
\hline $\mathrm{LDL}-\mathrm{c}, \mathrm{mmol} / \mathrm{L}$ & $3.51(2.83-4.20)$ & $3.74(2.96-4.41)$ & $3.86(2.96-4.72)^{a}$ & 0.039 \\
\hline $\mathrm{TG}, \mathrm{mmol} / \mathrm{L}$ & $1.11(0.85-1.41)$ & $1.43(1.03-2.01)^{a}$ & $2.27(1.73-2.91)^{a, b}$ & $<0.001$ \\
\hline AST, IU/L & $18.00(15.00-21.00)$ & $18.00(16.00-21.00)$ & $19.00(17.00-24.00)^{a, b}$ & $<0.001$ \\
\hline $\mathrm{ALT}, \mathrm{IU} / \mathrm{L}$ & $15.00(12.00-20.00)$ & $19.00(14.00-23.00)^{a}$ & $22.00(17.00-30.00)^{a, b}$ & $<0.001$ \\
\hline GGT, IU/L & $10.00(9.00-13.00)$ & $14.00(11.00-17.00)^{a}$ & $19.00(14.00-25.00)^{a, b}$ & $<0.001$ \\
\hline Uric acid, $\mu \mathrm{mol} / \mathrm{L}$ & 220.00 (191.00-261.00) & $256.00(225.00-298.00)^{a}$ & $316.00(254.00-355.00)^{a, b}$ & $<0.001$ \\
\hline Total bilirubin, $\mu \mathrm{mol} / \mathrm{L}$ & $7.50(5.70-9.80)$ & $7.10(5.80-9.80)^{a}$ & $6.60(5.10-8.60)^{a}$ & 0.011 \\
\hline $\mathrm{HsCRP}, \mathrm{mg} / \mathrm{L}$ & $0.52(0.30-1.15)$ & $1.36(0.89-2.40)^{a}$ & $2.16(1.16-4.24)^{a, b}$ & $<0.001$ \\
\hline Creatinine, $\mu \mathrm{mol} / \mathrm{L}$ & $58.00(53.00-64.00)$ & $63.00(57.00-71.00)^{a}$ & $65.00(58.00-73.00)^{a, b}$ & $<0.001$ \\
\hline FLI & $12.00(7.00-21.00)$ & $42.00(37.00-49.00)^{a}$ & $83.00(69.00-91.00)^{a, b}$ & $<0.001$ \\
\hline
\end{tabular}

Data are presented as median (interquartile range) and compared by Kruskal-Wallis with post hoc test.

a - significantly different from the first FLI group, $\mathrm{p}<0.05$; $\mathrm{b}$ - significantly different from the second FLI group, $\mathrm{p}<0.05$

HDL-c-High density lipoprotein cholesterol; LDL-c-Low density lipoprotein cholesterol; TG - Triglycerides; AST - Aspartate aminotransferase; ALT - Alanine aminotransferase; GGT - Gamma-glutamyl transferase; HsCRP - High-sensitivity C-reactive protein; FLI - Fatty liver index

Table V Spearman's correlation coefficients of FLI and other clinical parameters in men and women.

\begin{tabular}{|l|c|c|c|c|}
\hline & \multicolumn{2}{|c|}{ Men } & \multicolumn{2}{c|}{ Women } \\
\hline Variable & $\rho$ & $P$ & 0.315 & $<$ \\
\hline Age, $y$ ears & 0.165 & 0.009 & 0.894 & $<0.001$ \\
\hline BMl, $\mathrm{kg} / \mathrm{m}^{2}$ & 0.839 & $<0.001$ & 0.904 & $<0.001$ \\
\hline $\mathrm{WC}, \mathrm{cm}$ & 0.815 & $<0.001$ & 0.243 & $<0.001$ \\
\hline $\mathrm{SBP}, \mathrm{mmHg}$ & 0.097 & 0.131 & 0.148 & 0.001 \\
\hline DBP, $\mathrm{mmHg}$ & 0.030 & 0.643 & 0.547 & $<0.001$ \\
\hline Glucose, $\mathrm{mmol} / \mathrm{L}$ & 0.194 & 0.002 & 0.101 & 0.022 \\
\hline Total cholesterol, mmol/L & 0.112 & 0.080 & -0.562 & $<0.001$ \\
\hline HDL-c, mmol/L & -0.397 & $<0.001$ & 0.089 & 0.042 \\
\hline LDL-c, $\mathrm{mmol} / \mathrm{L}$ & 0.071 & 0.267 & 0.654 & $<0.001$ \\
\hline TG, $\mathrm{mmol} / \mathrm{L}$ & 0.593 & $<0.001$ & 0.165 & $<0.001$ \\
\hline AST, IU/L & 0.120 & 0.060 & 0.395 & $<0.001$ \\
\hline ALT, IU/L & 0.322 & $<0.001$ & 0.580 & $<0.001$ \\
\hline GGT, IU/L & 0.564 & $<0.001$ & 0.529 & $<0.001$ \\
\hline Uric acid, $\mu \mathrm{mol} / \mathrm{L}$ & 0.186 & 0.003 & -0.146 & 0.001 \\
\hline Total bilirubin, $\mu \mathrm{mol} / \mathrm{L}$ & -0.041 & 0.522 & 0.571 & $<0.001$ \\
\hline HsCRP, mg/L & 0.347 & $<0.001$ & 0.291 & $<0.001$ \\
\hline Creatinine, $\mu \mathrm{mol} / \mathrm{L}$ & -0.027 & 0.676 & & \\
\hline
\end{tabular}

Data are presented as correlation coefficient Rho $(\rho)$

BMI - Body mass index; WC - Waist circumference; SBP - Systolic blood pressure; DBP - Diastolic blood pressure; HDL-c - High density lipoprotein cholesterol; LDL-c - Low density lipoprotein cholesterol; TG -Triglycerides; AST - Aspartate aminotransferase; ALT - Alanine aminotransferase; GGT - Gamma-glutamyl transferase; HsCRP - High-sensitivity C-reactive protein 
Table VI Odds ratios (OR) after univariate and multivariate logistic regression analysis for ALT and UA predicting abilities towards fatty liver disease in men and women.

\begin{tabular}{|l|c|c|c|}
\hline $\begin{array}{l}\text { Predictors } \\
\text { Men }\end{array}$ & $\begin{array}{c}\text { Unadjusted } \\
\text { OR (95\%Cl) }\end{array}$ & $P$ & $\begin{array}{c}\text { Nagelkerke } \\
R^{2}\end{array}$ \\
\hline ALT, IU/L & $1.178(1.079-1.287)$ & $<0.001$ & 0.247 \\
\hline Uric acid, mmol/L & $1.004(0.998-1.010)$ & 0.170 & 0.021 \\
\hline Model 1 & $\begin{array}{c}\text { Adjusted } \\
\text { OR (95\%Cl) }\end{array}$ & $P$ & $\begin{array}{c}\text { Nagelkerke } \\
R^{2}\end{array}$ \\
\hline ALT, IU/L & $1.313(1.135-1.518)$ & $<0.001$ & 0.554 \\
\hline Uric acid, mmol/L & $1.008(0.999-1.017)$ & 0.089 & Nagelkerke \\
\hline $\begin{array}{l}\text { Predictors } \\
\text { Women }\end{array}$ & $\begin{array}{c}\text { Unadjusted } \\
\text { OR (95\%Cl) }\end{array}$ & $\mathrm{P}$ & 0.253 \\
\hline ALT, IU/L & $1.126(1.090-1.162)$ & 0.001 & 0.383 \\
\hline Uric acid, mmol/L & $1.020(1.015-1.029)$ & $<0.001$ & Nagelkerke \\
\hline Model 2 & $\begin{array}{c}\text { Adjusted } \\
\text { OR (95\%Cl) }\end{array}$ & $\mathrm{P}$ & 0.797 \\
\hline ALT, IU/L & $1.167(1.080-1.261)$ & $<0.001$ & 0.791 \\
\hline Model 3 & $1.014(1.007-1.021)$ & $<0.001$ & \\
\hline Uric acid, mmol/L & & & 0.818 \\
\hline Model 4 & $1.170(1.078-1.270)$ & $<0.001$ & \\
\hline ALT, IU/L & $1.014(1.006-1.022)$ & $<0.001$ & \\
\hline Uric acid, mmol/L & & & \\
\hline
\end{tabular}

Model 1: Age, Glucose, HDL-c, uric acid, hsCRP, ALT (all continuous variables) and antihyperlipemics and type 2 diabetes mellitus (all categorical variables)

Model 2: Age, Glucose, total cholesterol, HDL-c, LDL-c, ALT, creatinine, hsCRP, SBP, DBP, AST, total bilirubin (all continuous variables) and gender, antihyperglycemics, insulin, antihyperlipidemics, antihypertensives, type 2 diabetes mellitus (all categorical variables)

Model 3: Age, Glucose, total cholesterol, HDL-c, LDL-c, uric acid, creatinine, hsCRP, SBP, DBP, AST, total bilirubin (all continuous variables) and gender, antihyperglycemics, insulin, antihyperlipidemics, antihypertensives, type 2 diabetes mellitus (all categorical variables)

Model 4: Age, Glucose, total cholesterol, HDL-c, LDL-c, ALT, uric acid, creatinine, hsCRP, SBP, DBP, AST, total bilirubin (all continuous variables) and gender, antihyperglycemics, insulin, antihyperlipidemics, antihypertensives, type 2 diabetes mellitus (all categorical variables)

groups had significantly different BMI and WC because those variables were used for FLI calculation. The second and the third FLI group had significantly higher SBP and DBP than the first FLI group. Also, the third FLI group had significantly higher percentage of subjects on antihyperlipidemic therapy. The same percentages of men with DM2 were included in the first and in the third group, and both being higher than those in the second FLI group.

It was evident in Table /I that significantly higher percentage of women on antihyperglycemic, insulin, antihyperlipidemic and antihypertensive therapies and women with DM2 were in the third FLI group (i.e., group with fatty liver disease). Women in the second and in the third FLI group were older and had significantly higher DBP than women in the first group. Furthermore, BMI, WC and SBP were significantly different between all three groups, being higher in the second and the third group than in the first one.

Beside TG concentration and GGT, ALT activity was different between all men's FLI groups, being the highest in men with fatty liver disease (i.e., FLI $\geq 60$ ).
Also, HDL-c was the lowest in men with fatty liver disease (i.e., $F L I \geq 60$ ). Vice versa was established for hsCRP levels, which were the highest in men with FLI $\geq 60$. Total cholesterol concentration was the lowest in men without fatty liver disease (i.e., $\mathrm{FLI}<30$ ), (Table III).

As in men, TG concentration and GGT activity, were significantly different between all three FLI groups, being the highest in women with fatty liver. The same was found for glucose, HDL-c, uric acid, hsCRP, creatinine concentrations and ALT activity. Total cholesterol and LDL-c were higher in women with fatty liver disease than in those who did not have it. Total bilirubin concentration was the highest in women without fatty liver (i.e., FLI <30) and AST activity was the highest in women with fatty liver (i.e., FLI $\geq 60$ ), (Table IV).

Bivariate associations were revealed by Spearman's correlation analysis. In men, FLI was highly associated with all the parameters used for its calculation (BMI, WC, TG and GGT). Also, significant positive associations were established between FLI and age, glucose, ALT, uric acid, hsCRP and signifi- 
Table VII ROC analysis for single parameter discriminatory abilities towards fatty liver disease development in men and women.

\begin{tabular}{|c|c|c|c|c|c|}
\hline Predictors & $\mathrm{AUC}(95 \% \mathrm{Cl})$ & SE & Sensitivity (\%) & Specificity (\%) & $\mathrm{P}$ \\
\hline \multicolumn{6}{|l|}{ Men } \\
\hline $\mathrm{ALT}, \mathrm{IU} / \mathrm{L}$ & $0.804(0.719-0.890)$ & 0.044 & 61 & 95 & $<0.001$ \\
\hline Uric acid, $\mu \mathrm{mol} / \mathrm{L}$ & $0.576(0.500-0.650)$ & 0.067 & 74 & 46 & $>0.05$ \\
\hline Model 1 & $0.932(0.891-0.974)$ & 0.021 & 74 & 100 & 0.001 \\
\hline \multicolumn{6}{|l|}{ Women } \\
\hline$A L T, I U / L$ & $0.746(0.698-0.794)$ & 0.025 & 63 & 72 & $<0.001$ \\
\hline Uric acid, $\mu \mathrm{mol} / \mathrm{L}$ & $0.820(0.779-0.858)$ & 0.021 & 65 & 84 & $<0.001$ \\
\hline Model 2 & $0.965(0.948-0.982)$ & 0.009 & 90 & 91 & $<0.001$ \\
\hline Model 3 & $0.965(0.948-0.982)$ & 0.009 & 88 & 92 & $<0.001$ \\
\hline Model 4 & $0.971(0.956-0.986)$ & 0.008 & 92 & 90 & $<0.001$ \\
\hline
\end{tabular}

Model 1: Age, Glucose, HDL-c, uric acid, hsCRP, ALT (all continuous variables) and antihyperlipidemics and type 2 diabetes mellitus (all categorical variables)

Model 2: Age, Glucose, total cholesterol, HDL-C, LDL-c, ALT, creatinine, hsCRP, SBP, DBP, AST, total bilirubin (all continuous variables) and gender, antihyperglycemics, insulin, antihyperlipidemics, antihypertensives, type 2 diabetes mellitus (all categorical variables)

Model 3: Age, Glucose, total cholesterol, HDL-C, LDL-c, uric acid, creatinine, hsCRP, SBP, DBP, AST, total bilirubin (all continuous variables) and gender, antihyperglycemics, insulin, antihyperlipidemics, antihypertensives, type 2 diabetes mellitus (all categorical variables)

Model 4: Age, Glucose, total cholesterol, HDL-c, LDL-c, ALT, uric acid, creatinine, hsCRP, SBP, DBP, AST, total bilirubin (all continuous variables) and gender, antihyperglycemics, insulin, antihyperlipidemics, antihypertensives, type 2 diabetes mellitus (all categorical variables)

cant negative association between FLI and HDL-c. In women, FLI correlated positively with all the examined parameters, except with HDL-c and total bilirubin. FLI correlated highly negatively with HDL-c and total bilirubin. Correlation coefficients $(\rho)$ and $P$ values were presented in Table $\mathrm{V}$.

Further statistical analysis included binary logistic regression in order to determine whether measurement of ALT and uric acid which significantly correlated with FLI could have a potential predictive role on fatty liver occurrence. The first FLI group (FLI $<30$ ) was selected as a reference group and coded as 0 while the third FLI group (FLI $\geq 60$ ) was selected as group of patients with fatty liver disease and coded as 1. Coding was performed separately in men and women. Unadjusted analysis indicated that in men ALT was a predictor and in women ALT and uric acid were predictors for fatty liver disease. As ALT activity rose for $1 \mathrm{IU} / \mathrm{L}$, probability for fatty liver disease increased by $17.8 \%$ in men (Table VI). The unadjusted Nagelkerke $\mathrm{R}^{2}$ for FLI of 0.247 means that $24.7 \%$ of variation in FLI was caused by ALT in men. As ALT activity rose for $1 \mathrm{IU} / \mathrm{L}$ and uric acid concentration rose for $1 \mu \mathrm{mol} / \mathrm{L}$, probability for fatty liver disease in women increased by $12.6 \%$ and $2 \%$, respectively (Table $\mathrm{VI}$ ). In women, the unadjusted Nagelkerke $\mathrm{R}^{2}$ for $\mathrm{FLI}$ of 0.253 and 0.383 means that $25.3 \%$ and $38.3 \%$ of variations in FLI were caused by ALT and uric acid, respectively.

Thereafter, we constructed logistic regression models to further test the potential independent associations of the ALT and uric acid with fatty liver disease in men and women. The best models for each of the investigated clinical parameter are presented in Table VI. In men the Model 1 incorporated adjustments for all the clinical parameters significantly correlated with FLI as continuous variables, and therapy usage and DM2 presence differently distributed among FLI groups as categorical variables. In women models 2, 3 and 4 incorporated adjustments for all the clinical parameters significantly correlated with $\mathrm{FLI}$ as continuous variables and therapy usages and DM2 presence differently distributed among FLI groups as categorical variables. ALT kept its independent predictive power on fatty liver disease in men [OR $(95 \% \mathrm{Cl})=1.313(1.135-$ 1.518), $P<0.001]$. The level of influence of the Model 1 on variation of FLI was $55.4 \%$ (Table VI). Also, ALT kept its independent prediction in Model 2 and Model 4 on fatty liver disease in women: [OR $(95 \% \mathrm{Cl})=1.167$ (1.080-1.261), $\mathrm{P}<0.001]$ and [OR $(95 \% \mathrm{Cl})=1.170(1.078-1.270), \mathrm{P}<0.001]$, respectively. Uric acid was independent predictor of fatty liver disease only in women: the Model 3 [OR $(95 \% \mathrm{Cl})=1.014(1.007-1.021), \mathrm{P}<0.001]$ and the Model 4 [OR $(95 \% \mathrm{Cl})=1.014$ (1.006-1.022), $\mathrm{P}<0.001]$. The levels of influence of the Models 2, 3 and 4 on variation of $\mathrm{FLI}$ were $79.9 \%, 79.1 \%$ and $81.8 \%$, respectively.

Furthermore, we performed the ROC analysis in order to test the clinical accuracy of ALT in men and ALT and uric acid in women towards the presence of fatty liver disease (Table VII). The calculated AUC for ALT as a single parameter indicated its good clinical accuracy (23): AUC $(95 \% \mathrm{Cl})=0.804$ (0.719$0.890), P<0.001$ in development of fatty liver 


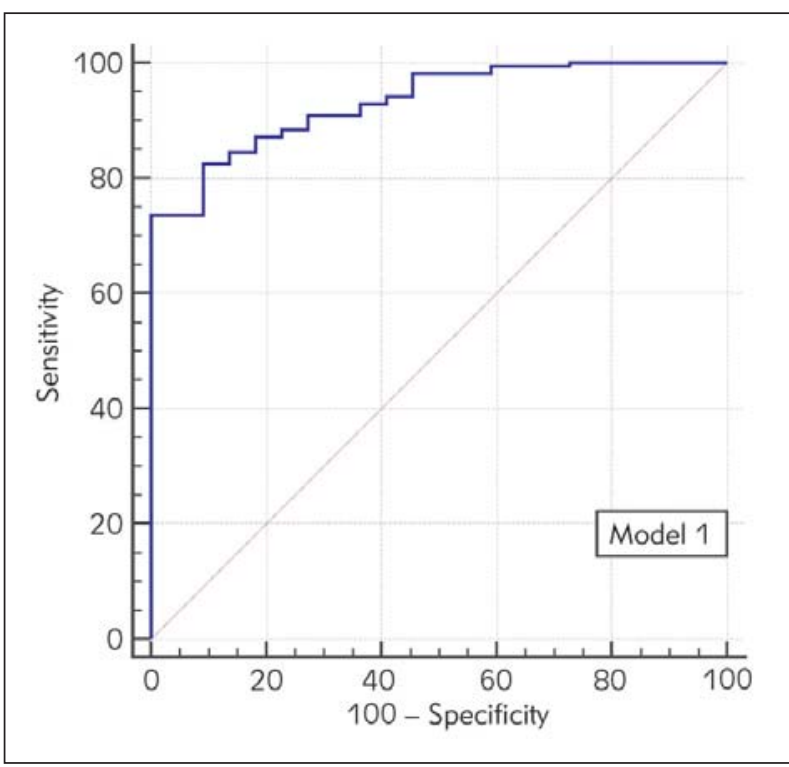

Figure 1 ROC curves for models' discriminatory abilities towards fatty liver disease in men.

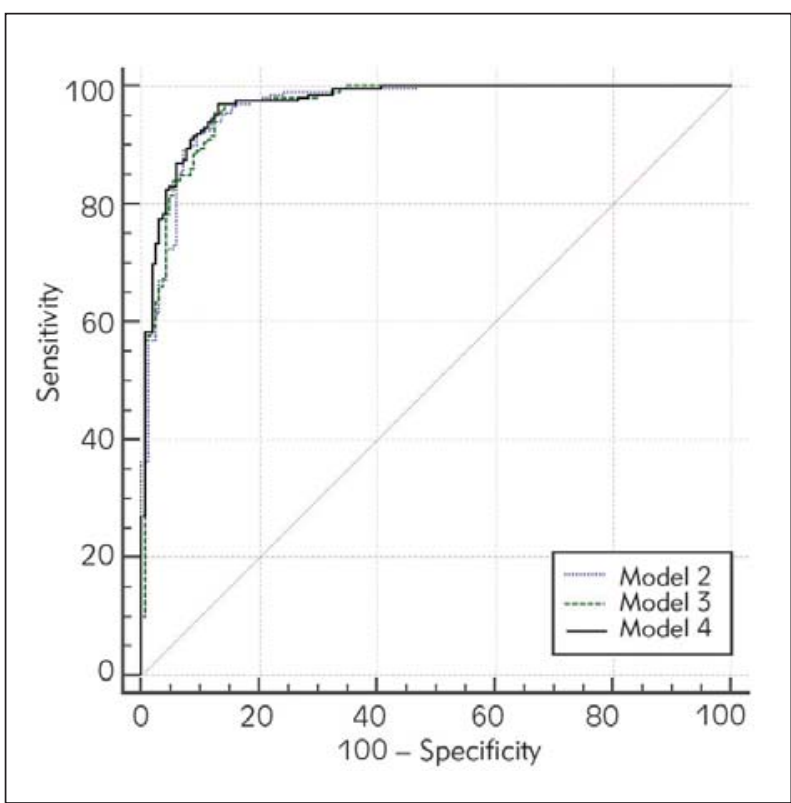

Figure 2 ROC curves for models' discriminatory abilities towards fatty liver disease in women.

disease in men. The ROC-analysis-estimated clinical accuracy of uric acid in men as a single parameter did not show any significant discriminatory ability towards fatty liver disease: AUC $(95 \% \mathrm{Cl})=0.576(0.500$ $0.650), P>0.05$. The calculated AUC for ALT and uric acid in women as single parameters revealed that only uric acid had a good clinical accuracy for fatty liver development: AUC $(95 \% \mathrm{Cl})=0.820(0.779$ 0.858), $\mathrm{P}<0.001$ (Table VII).

Additionally, we constructed four models using predictive probabilities generated by logistic regression analysis. Figure 1 and Table VII showed
ROC curve for the Model 1 in men. The AUC of this model was 0.932 indicated excellent discriminatory capability towards fatty liver development. The same was found for models 2, 3 and 4 generated in women (Figure 2 and Table VII). All of them indicated excellent discriminatory capability towards fatty liver development. The pair-wise comparisons of ROC curves between Models 2 and 4 (AUC difference $=0.006, P=0.073)$ and Models 3 and $4(\mathrm{AUC}=0.006$, $\mathrm{P}=0.061)$ showed that improvement with ALT and uric acid together in the same model was not statistically significant in discriminatory capability towards fatty liver disease (data not presented). Also, a pair-wise comparison of Model 2 and Model 3 ROC curves (AUC difference $=0.000, P=0.956$ ) was not significant in discriminating patients with and without fatty liver disease. ALT in the Model 2 and uric acid in the Model 3, both with the same other clinical parameters could be considered to have the same clinical accuracy for fatty liver disease development.

Finally, ROC analysis was used to estimate cutoff values for ALT in men and ALT and uric acid in women in our study population associated with increase in the prevalence of the fatty liver disease. The cut-off value for ALT was 19 IU/L (AUC $=0.746$, sensitivity $63 \%$, specificity $72 \%, \mathrm{P}<0.001)$ in women and $22 \mathrm{IU} / \mathrm{L} \quad(A \cup C=0.804$, sensitivity 61\%, specificity $95 \%, P<0.001)$ in men. The cut-off value for uric acid was $274 \mu \mathrm{mol} / \mathrm{L} \quad$ (AUC $=0.821$, sensitivity $68 \%$, specificity $82 \%, P<0.001$ ) in women.

\section{Discussion}

The findings of the current study reveal that ALT was independent predictor of the fatty liver disease as determined by $\mathrm{FLI}$ in both men and women, whereas serum uric acid was its independent predictor only in women (Table VI). Moreover, unlike commonly used threshold of $40 \mathrm{IU} / \mathrm{L}$ in clinical practice (12), we have shown that lower cut-off levels of ALT activity in our study population are associated with the increased prevalence of the fatty liver disease. Namely, the cutoff values in our study are as follows: ALT was 19 IU/L (AUC $=0.746$, sensitivity $63 \%$, specificity $72 \%$, $\mathrm{P}<0.001)$ in women and $22 \mathrm{IU} / \mathrm{L} \quad(\mathrm{AUC}=0.804$, sensitivity $61 \%$, specificity $95 \%, \mathrm{P}<0.001$ ) in men. Our results are in line with some previous reports that suggest the ALT cut-off level used in NAFLD diagnosis should be downward in order to improve the sensitivity of the method and to better identify individuals being at risk of NAFLD, as well as to prevent its progression $(11,12)$. Namely, Miyake et al. (11) have reported ALT activity to be $17 \mathrm{IU} / \mathrm{L}$ for females and $25 \mathrm{IU} / \mathrm{L}$ for males, as the cut-off levels used for NAFLD diagnosis. Epidemiological studies have also recommended lower cut-off levels (i.e., 19 IU/L for females and $30 \mathrm{IU} / \mathrm{L}$ for males) to be accepted as a normal upper limit level (12). 
However, despite suggested lower ALT cut-off levels, there are studies that reject that $A L T$ is a reliable marker of NAFLD $(8,12,13)$. In line with this, van den Berg et al. (8) have shown that even $80.3 \%$ of individuals that were classified into the group with NAFLD had normal ALT, when using the upper limit of normal levels of this enzyme. Similarly, another study has confirmed that normal ALT activities were observed in $79 \%$ of subjects with hepatic steatosis (24). Although ALT exists in two isoforms in humans $(25,26)$, the ALT2 isoform makes the main contribution to homeostasis of free fatty acids (FFA), being highly expressed in adipose tissue of obese individuals (25). In addition, increased lipid peroxidation can cause the ALT to leak more easily out of the hepatocytes. Moreover, it is assumed that increased ALT activity in circulation may also be a result of the compensatory response to the impaired hepatic insulin signalling (25).

We failed to confirm the independent relationship between uric acid and FLI in males, but confirmed only in females. In addition, the cut-off value for uric acid was $274 \mu \mathrm{mol} / \mathrm{L} \quad(\mathrm{AUC}=0.821$, sensitivity $68 \%$, specificity $82 \%, P<0.001$ ) in women. However, it is questionable whether hyperuricemia raises the risk for fatty liver occurrence in females more than in males since controversy exists in literature concerning the relationship between serum uric acid and NAFLD (14-17). A study conducted by Wu et al. (15) in a large population group showed that the relationship between uric acid and NAFLD was significantly stronger in females than in males. Similar observations were reported by Yang et al. (16) in a four-year retrospective cohort study in Chinese population. The independent effect of hyperuricemia (i.e., defined as serum uric acid level level of $>360 \mu \mathrm{mol} / \mathrm{L}$ in females) on NAFLD was stronger in females than in males, but it was found only in nonobese subjects (16). To make this question more complicated, Fan et al. (14) reported that an increase in serum uric acid was independently associated with the higher risk of NAFLD only in males, but not in females in a study that encompassed exclusively individuals with DM2.

On the contrary, recent large meta-analysis (17) has reported that increased risk for NAFLD occurrence is significantly associated with hyperuricemia in both males and females. Moreover, uric acid was found to directly inhibit insulin signalling and induce insulin resistance, which is considered to be the underlying mechanism of hepatic steatosis (14). Importantly, Lanaspa et al. (27) reported that serum uric acid could directly stimulate hepatic fat synthesis.

However, due to gender-difference observed in relation with serum uric acid and hepatic steatosis in many studies, it may be assumed that uric acid acts on fatty liver development through different mechanisms in males and females. Future studies are needed to elucidate this assumption.

The main disadvantage of the current study is its cross-sectional design, which does not allow making a cause-effect inference. Moreover, we have not evaluated hepatic steatosis directly by ultrasound, computed tomography, or liver biopsy, but with FLI. Nevertheless, we followed the 2016 EASL-EASDEASO NAFLD guidelines recommendations on the usage of the serum biomarkers as a preferred diagnostic tool with the FLI as one of the best validated steatosis scores for screening studies in large population samples (22). The strength of the current study, and advantage over our previous studies $(20,21)$ is the fact that we included a large sample size of population of Montenegro. Therefore, we assume that lower cut-off levels of ALT in both males and females, as well as serum uric acid levels in females only, can be reliable predictors of FLI, which can facilitate identifying individuals having a great risk of fatty liver disease development. Additional studies are needed to confirm our results, as well as to make a comparison between these data (i.e., fatty liver assessed with FLI algorithm) and fatty liver as assessed with ultrasound in relation to ALT and uric acid levels.

Acknowledgement. This work was financially supported partially by a grant from the Ministry of Education, Science and Technological Development, Republic of Serbia (Project number 175035).

We thank all the examinees for their participation in the current study.

\section{Conflict of interest statement}

The authors stated that they have no conflicts of interest regarding the publication of this article. 


\section{References}

1. Hazlehurst JM, Woods C, Marjot T, Cobbold JF, Tomlinson JW. Non-alcoholic fatty liver disease and diabetes. Metabolism 2016; 65(8): 1096-108.

2. Liu H, Lu HY. Non-alcoholic fatty liver disease and cardiovascular disease. World J Gastroenterol 2014; 20(26): 8407-15.

3. Miyake T, Yoshida S, Furukawa S, Sakai T, Tada F, Senba $\mathrm{H}$, et al. Ipragliflozin Ameliorates Liver Damage in Non-alcoholic Fatty Liver Disease. Open Med (Wars). 2018; 13: 402-9.

4. Masugi $Y$, Abe T, Tsujikawa $H$, Effendi $K$, Hashiguchi $A$, Abe $M$, et al. Quantitative assessment of liver fibrosis reveals a nonlinear association with fibrosis stage in non-alcoholic fatty liver disease. Hepatol Commun 2017; 2(1): 58-68.

5. Bedogni G, Bellentani S, Miglioli L, Masutti F, Passalacqua $M$, Castiglione $A$, et al. The Fatty Liver Index: a simple and accurate predictor of hepatic steatosis in the general population. BMC Gastroenterol 2006; 6: 33.

6. Zelber-Sagi S, Webb M, Assy N, Blendis L, Yeshua $H$, Leshno $M$, et al. Comparison of fatty liver index with non-invasive methods for steatosis detection and quantification. World J Gastroenterol 2013; 19(1): 5764.

7. Zhang S, Du T, Zhang J, Lu H, Lin X, Xie J, et al. The triglyceride and glucose index (TyG) is an effective biomarker to identify non-alcoholic fatty liver disease. Lipids Health Dis 2017; 16: 15.

8. Van den Berg EH, Amini M, Schreuder TCMA, Dullaart RPF, Faber KN, Alizadeh BZ, et al. Prevalence and determinants of non-alcoholic fatty liver disease in lifelines: A large Dutch population cohort. PLoS ONE 2017; 12(2): e0171502.

9. Huang Q, Yu J, Zhang X, Liu S, Ge Y. Association of the serum uric acid level with liver histology in biopsyproven non-alcoholic fatty liver disease. Biomed Rep 2016; 5(2): 188-92.

10. Liu J, Xu C, Ying L, Zang S, Zhuang Z, Lv H, et al. Relationship of serum uric acid level with non-alcoholic fatty liver disease and its inflammation progression in non-obese adults. Hepatol Res 2017; 47(3): E104E112.

11. Miyake T, Kumagi T, Hirooka M, Koizumi M, Furukawa $\mathrm{S}$, Ueda $\mathrm{T}$, et al. Metabolic markers and ALT cut-off level for diagnosing non-alcoholic fatty liver disease: a community-based cross-sectional study. J Gastroenterol 2012; 47(6): 696-703.

12. Bril F, Cusi K. Management of Non-alcoholic Fatty Liver Disease in Patients With Type 2 Diabetes: A Call to Action. Diabetes Care 2017; 40(3): 419-30.

13. Foghsgaard S, Andreasen C, Vedtofte L, Andersen ES, Bahne E, Strandberg C, et al. Non-alcoholic Fatty Liver Disease Is Prevalent in Women With Prior Gestational Diabetes Mellitus and Independently Associated With Insulin Resistance and Waist Circumference. Diabetes Care 2017; 40(1): 109-16.
14. Fan N, Zhang L, Xia Z, Peng L, Wang Y, Peng Y. SexSpecific Association between Serum Uric Acid and Non-alcoholic Fatty Liver Disease in Type 2 Diabetic Patients. J Diabetes Res 2016; 2016: 3805372.

15. Wu SJ, Zhu GQ, Ye BZ, Kong FQ, Zheng ZX, Zou H, et al. Association between sex-specific serum uric acid and non-alcoholic fatty liver disease in Chinese adults: a large population-based study. Medicine (Baltimore) 2015; 94(17): e802.

16. Yang C, Yang S, Xu W, Zhang J, Fu W, Feng C. Association between the hyperuricemia and nonalcoholic fatty liver disease risk in a Chinese population: A retrospective cohort study. PLoS One 2017; 12(5): e0177249.

17. Gong S, Song J, Wang L, Zhang S, Wang Y. Hyperuricemia and risk of non-alcoholic fatty liver disease: a systematic review and meta-analysis. Eur J Gastroenterol Hepatol 2016; 28(2): 132-8.

18. Klisic AN, Vasiljevic ND, Simic TP, Djukic TI, Maksimovic MZ, Matic MG. Association between Creactive protein, anthropometric and lipid parameters among healthy normal weight and overweight postmenopausal women in Montenegro. Lab Med 2014; 45(1): 12-6.

19. Klisic A, Kavaric N, Jovanovic M, Zvrko E, Skerovic V, Scepanovic A, et al. Association between unfavorable lipid profile and glycemic control in patients with type 2 diabetes mellitus. J Res Med Sci 2017; 22: 122.

20. Klisic A, Isakovic A, Kocic G, Kavaric N, Jovanovic M, Zvrko E, et al. Relationship between Oxidative Stress, Inflammation and Dyslipidemia with Fatty Liver Index in Patients with Type 2 Diabetes Mellitus. Exp Clin Endocrinol Diabetes 2018; 126(06): 371-8.

21. Klisic A, Kavaric N, Jovanovic M, Soldatovic I, Gligorovic-Barhanovic N, Kotur-Stevuljevic J. Bioavailable testosterone is independently associated with fatty liver index in postmenopausal women. Arch Med Sci 2017; 5(13): 1188-96.

22. European Association for the Study of the Liver (EASL). Electronic address: easloffice@easloffice.eu, European Association for the Study of Diabetes (EASD), European Association for the Study of Obesity (EASO). EASLEASD-EASO Clinical Practice Guidelines for the management of non-alcoholic fatty liver disease. J Hepatol 2016; 64(6): 1388-402.

23. Milinković N, Ignjatović S, Šumarac Z, Majkić-Singh N. Uncertainty of measurement in laboratory medicine. J Med Biochem 2018; 37: 279-88.

24. Browning JD, Szczepaniak LS, Dobbins R, Nuremberg P, Horton JD, Cohen JC, et al. Prevalence of hepatic steatosis in an urban population in the United States: impact of ethnicity. Hepatology 2004; 40(6): 138795.

25. Aliasghari F, Izadi A, Jabbari M, Imani B, Gargari PB, Asjodi F, Ebrahimi S. Are vaspin and omentin-1 related to insulin resistance, blood pressure and inflammation in NAFLD patients? J Med Biochem 2018: 470-475. 
26. Ünüvar S, Tanrıverdi Z, Aslanhan H. Potential prognostic role of immune system activation marker neopterin in patients with type 2 diabetes. J Med Biochem 2018, 37; 465-9.
27. Lanaspa MA, Sanchez-Lozada LG, Choi YJ, Cicerchi C, Kanbay M, Roncal-Jimenez CA, et al. Uric acid induces hepatic steatosis by generation of mitochondrial oxidative stress: potential role in fructose-dependent and -independent fatty liver. J Biol Chem 2012; 287: 40732-44.

Received: December 12, 2018

Accepted: January 5, 2019 